\title{
Baseline wander Removal in ECG using an efficient method of EMD in combination with wavelet
}

\author{
Abdul Qayoom Bhat \\ Department of Electronics and communication Engineering, Lovely Professional University Punjab India
}

\begin{abstract}
ECG signal processing is a challenging field which has to deal with several issues. ECG, electrocardiogram plays a vital role in the diagnosis of heart related problems. Good quality ECG is used by the doctors for identification of physiological and pathological phenomena. ECG is very sensitive in nature and even if small amount of noise interferes with it, the characteristics of the signal change. Therefore in order to have a better understanding of thc cardiological phenomenon a lot of denoising algorithms have been proposed for removal of artifacts from ECG, like FIR, IIR or wavelet filtering. Each method suffers with some limitations. Here a new method is proposed which combines EMD with wavelet. On applying EMD along with WT, we get better results.This method, to some extent minimizes the limitations of EMD alone or wavelet alone.
\end{abstract}

Keywords: ECG, Baseline wander, Wavelet Transform, EMD.

\section{INTRODUCTION}

The ECG is a record of electric potentials generated by different actions of heart. These different movements of heart are called as depolarization and repolarisation. The depolarization refers tothe contraction and repolarisation refers to the resting state of heart. The depolarization and repolarisation occurs as a result of movement of $\mathrm{Na}+, \mathrm{K}+$ and $\mathrm{Cl}$ - ions through the semi permeable cell membranes[1]. The ECG signal is typically in the range of $2 \mathrm{mV}$ and requires a recording bandwidth of 0.1 to $150 \mathrm{~Hz}$. This signal is achieved by placing electrodes at different positions on the patient's body. Clinical ECGs are obtained by a standard 12 lead system. The ECG signal and heart rate reflects the cardiac health of human heart. Any disorder in heart rate or rhythm or change in the morphological pattern of ECG signal is an indication of cardiac arrhythmia. Therefore it is very important that the ECG signal obtained from a patient is not corrupted by noise as it becomes very difficult for a specialist to judge the ECG signal if artifacts(noise) are present in the signal[2]

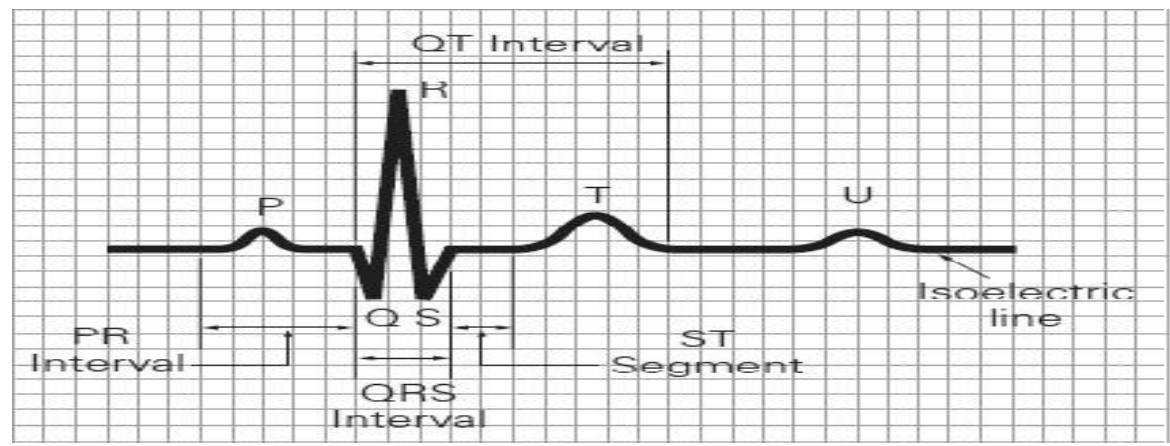

Fig 1. ECG Waveform

The major sources of noise which effect the ECG signal are: Power line interference, Baseline wandering, Electrode contact noise, Motion Artifacts, Muscle Artifacts, Noise generated by electronic devices used in signal processing circuits, Electrical interference external to the subject and recording system.

Baseline wandering mainly occurs due to movement of the subject (or patient), change in the electrode resistance due to perspiration, or due to respiration. The base line wandering causes faulty measurements of the signal features. Usually the base line wandering has the same frequency content as that of $\mathrm{T}$ wave. However due to certain tests such as the stress test, the baseline wandering may vary unpredictably. ST segment measurements are strongly affected due to base line wandering. 


\subsection{FIR and IIR filtering}

\section{BASIC DENOISING METHODS}

Digital filters are either Finite Impulse Response (FIR) filters or Infinite Impulse response (IIR) filters, depending on the impulse response of the system i-e; output of the system when a unit impulse is given at the input. These are characterized by the following equation(1):

$$
H(z)=\frac{B(z)}{A(z)}=\frac{b_{0}+b_{1} z^{-1}+b_{2} z^{-2}+\ldots \ldots+b_{n} z^{-n}}{1+a_{1} z^{-1}+a_{2} z^{-2}+\ldots \ldots+a_{m} z^{-m}}
$$

If all the coefficients (a's) in the denominator are made zero, the resulting filter will be FIR filter. Thus equation of this filter will be equation (2):

$$
y(n)=\sum_{k=0}^{M} b_{k} x(n-k)
$$

The output of FIR filter depends only upon present and previous inputs. As we can see that thisfilter has no feedback and hence its output does not depend on previous outputs. IIR filters have the following difference equation (3):

$$
y(n)=-\sum_{k=1}^{N} a_{k} y(n-k)+\sum_{k=1}^{M} b_{k} x(n-k)
$$

In this case the filter output depends upon previous inputs, present inputs and also on previous outputs. IIR filters are useful for high-speed designs because they typically require a lower number of multiply compared to FIR filters[3].

The limitations of this type of filtering are:

- FIR filters remove the baseline noise of ECG signal at the expense of some ringing effect at the starting of waveform.

- These are static filters i-e they have fixed filter coefficients. Since ECG is a non stationary signal, the characteristics of signal change with time, so every time for a new signal we have to design a new filter.

- It increases the memory requirement for FIR filters.

\subsection{Wavelet Denoising}

The wavelet transform is similar to the Fourier transform. For the FFT, the basis functions are sines and cosines. For the wavelet transform, the basis functions are more complicated called wavelets or analyzing wavelets. In wavelet analysis, the signal is broken into shifted and scaled versions of the original (or mother) wavelet. The fact that wavelet transform is a multiresolution analysis makes it very suitable for analysis of nonstationary signals such as the ECG signal [4].

The Fourier transform is useful tool to analyze the frequency components of the signal. However, if we take the Fourier transform over the whole time axis, we cannot tell at what instant a particular frequency rises. Short-time Fourier transform (STFT) uses a sliding window to find spectrogram, which gives the information of both time and frequency. But still another problem exists i.e. the length of window limits the resolution in frequency. Wavelet transform seems to be a solution to the problem above. Wavelet transforms (WT) are based on small wavelets with limited duration. In WT both the time and frequency resolutions vary in time-frequency plane in order to obtain a multiresolution analysis.

\subsection{Wavelets}

In comparison to the Fourier transform, the analyzing function of the wavelet transform can be chosen with more freedom, without the need of using sine-forms. A wavelet function $\Psi(\mathrm{t})$ is a small wave, which must be oscillatory in some way to discriminate between different frequencies [5]. The wavelet contains both the analyzing shape and the window. For the CWT several kind of wavelet functions are developed which all have specific properties [5], [6].

An analyzing function $\Psi(\mathrm{t})$ is classified as a wavelet if the following mathematical criteria are satisfied [5]:

1. A wavelet must have finite energy, equation (4). 


$$
\mathrm{E}=\int_{-\infty}^{\infty}|\Psi(\mathrm{t})|^{2} \mathrm{dt}<\infty
$$

The energy E equals the integrated squared magnitude of the analyzing function $\Psi(t)$ and must be less than infinity, equation (5).

$$
\mathrm{E}=\int_{-\infty}^{\infty}|\Psi(\mathrm{t})|^{2} \mathrm{dt}<\infty
$$

2. If $\Psi(\mathrm{f})$ is the Fourier transform of the wavelet $\Psi(\mathrm{t})$, the following condition must hold, equation (6).

$$
C_{\Psi}=\int_{0}^{\infty} \frac{|\Psi(f)|^{2}}{f} d f<\infty
$$

This condition implies that the wavelet has no zero frequency component $(\Psi(0)=0)$, i.e. the mean of the wavelet $\Psi(\mathrm{t})$ must equal zero. This condition is known as the admissibility constant. The value of $C_{\Psi}$ depends on the chosen wavelet. For complex wavelets the Fourier transform $\Psi(\mathrm{f})$ must be both real and vanish for negative frequencies.

The wavelet transform decomposes the signal into approximate and detail information by expressing a signal $x(t)$ in terms of scaling function $\varphi j, k(t)$ and mother wavelet function $\psi j, k(t)$. Here $j$ is the parameter of dilation or the visibility in frequency and $k$ is the parameter of the position. Thus, it helps in analyzing the signal at different frequency bands with different resolutions[7].

\subsection{Reasons for wavelet filtering as not a best choice for ECG filtering:}

Wavelet filtering is a better method for ECG filtering but it still has some of the disadvantages because of which it can't be directly used for filtering of ECG signals. The disadvantages are:

- Although wavelet transforms are suitable for noise reduction, its pre-divided frequency feature limits its ability to decompose the signal into different frequencies according to the inherent characteristics of ECG signals.

- The hard thresholding may lead to oscillations of the reconstructed ECG signal.

- The soft thresholding may reduce the amplitude of $\mathrm{R}$ wave which is more important for diagnosis of cardiac arrhythmia.

\section{Empirical Mode Decomposition(EMD)}

A necessary condition to represent nonlinear and nonstationary data is to have an adaptive basis. An a priori defined function cannot be relied on as a basis, no matter how sophisticated the basis function might be. Empirical mode decomposition (EMD) method has offered a powerful method for nonlinear non-stationary data analysis. It is the first local and adaptive method in frequency-time analysis. Contrary to almost all the previous methods, this new method is intuitive, direct, a posteriori and adaptive, with the basis of the decomposition based on, and derived from, the data. The decomposition is based on the assumptions[8]:

(1) The signal has at least two extrema - one maximum and one minimum.

(2) The characteristic time scale is defined by the time lapse between the extrema.

(3) If the data were totally devoid of extrema but contained only inflection points, then it can be differentiated once or more times to reveal the extrema. Final results can be obtained by integration(s) of the components.

A new nonlinear technique, referred to as Empirical Mode Decomposition (EMD), has recently been pioneered by N.E. Huang et al. for adaptively representing nonstationary signals as sums of zero-mean AM-FM components [9]. This decomposition method is specific to the signal being analyzed. The method decomposes a signal into a sum of components, each with slowly varying amplitude and phase, which is called an Intrinsic Mode Function (IMF). The Empirical Mode Decomposition process separates the IMFs with decreasing order of frequency, i.e., it separates high frequency component first and the low frequency component at the end. Mathematical Expression of Empirical Mode Decomposition processed signal is defined as follows[10], equation (7):

$$
x(t)=\sum_{n-1}^{N} c_{n}(t)+r_{N}(t)
$$

Where $c_{n}(t)$ is Intrinsic Mode Function set and $r_{N}(t)$ is residual term. 
Baseline wander Removal in ECG using an efficient method of EMD in combination with wavelet.

\subsection{Intrinsic Mode Function (IMF)}

The Empirical Mode Decomposition process produces a set of IMFs that represent the original data vector broken down into frequency components from highest to lowest frequency. Lower order IMFs capture fast oscillation modes while higher order IMFs typically represent slow oscillation modes.If all of the IMFs for a given signal are added together, the resulting "summation" signal is a near perfect match for the original signal (i.e., with little or no leftover), yielding a high level of confidence in the EMD results. These IMFs are defined as functions that:

(1) have the same number of zero-crossings and extrema (or at most differ by one), and

(2) An IMF must have zero local mean.

In the second criterion, the definition of what constitutes the local mean is somewhat vague. For some $€$, we can write the requirement as:

$$
\overline{\operatorname{IMF}}_{\varepsilon}(t)=\frac{1}{\varepsilon} \int_{t-\frac{\varepsilon}{2}}^{t+\frac{\varepsilon}{2}} \operatorname{IMF}(r) d r=\mathrm{O}
$$

In practice, the value of $€$ depending upon application. The choice determines to some extent how finely the IMFs will be resolved by essentially determining a frequency above which fluctuations will be averaged out.

\section{Methodology}

When a noisy signal is decomposed using EMD, the high frequency noise components are mainly present in the initial IMFs. But the baseline wander noise is present in the last IMFs. In this work, an estimate of baseline wander is made using a bank of low pass filters. A particular value of variance with respect to mean is used to determine the noise order of IMFs. Since the bandwidth of ECG is usually in the range from 0.05 to 100 $\mathrm{Hz}$, the power spectrum of signal IMFs will be concentrated on a short range of frequencies. The spectrum of noisy IMFs will be relatively flat as compared to signal IMFs. The proposed noise removal method using EMD is as, the different steps are explained below:

Step1. The ECG signals are taken from MIT/BIH arrhythmia data base. Every file in the data base consists of two lead recordings sampled at $360 \mathrm{~Hz}$ sampling frequency with 11 bits per sample of resolution. The noisy signal $\mathrm{s}(\mathrm{t})$ is obtained as $\mathrm{s}(\mathrm{t})=\mathrm{x}(\mathrm{t})+\mathrm{n}(\mathrm{t})$ where $\mathrm{x}(\mathrm{t})$ is the original ECG and $\mathrm{n}(\mathrm{t})$ is the noise signal.

Step 2: The noisy ECG signal is decomposed into IMFs using EMD method.

Step 3: The number of noisy IMFs, $n$, is obtained using a bank of low pass filters.

Step 4: The noisy IMFs are filtered using wavelet transform, by selecting a proper threshold.

Step 5: After these IMFs are filtered, the signal is reconstructed by adding the filtered IMFs with noise free IMfs.

\section{Waveforms and Results}

Arbitrary ECG record is chosen from the MIT-BIH database as clean signal, and baseleine wander noise is added to it to generate noisy ECG. Then, the results of two methods are compared EMD-FIR and EMDWavelet. Denoising results are compared using the SNR of denoised signals. For obtaining the results Matlab 2010a is used.

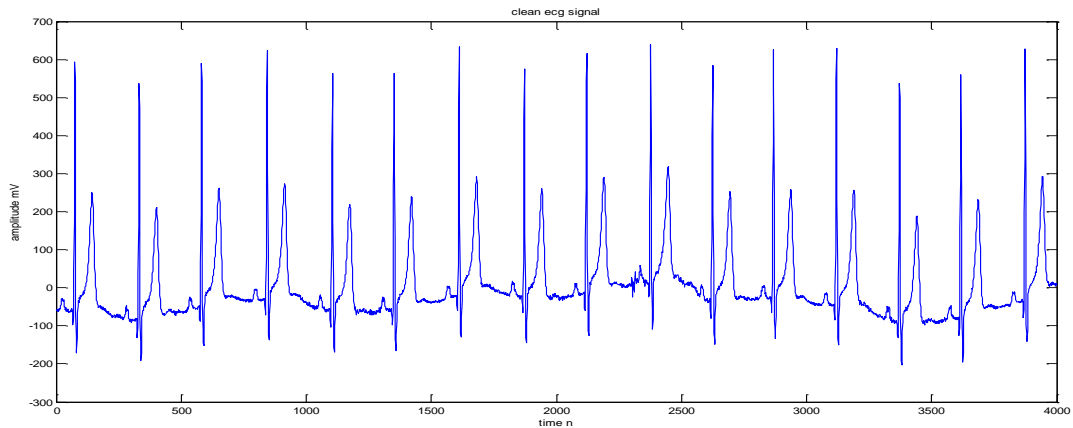

Fig 2 Aclean signal from MIT-BIH database 


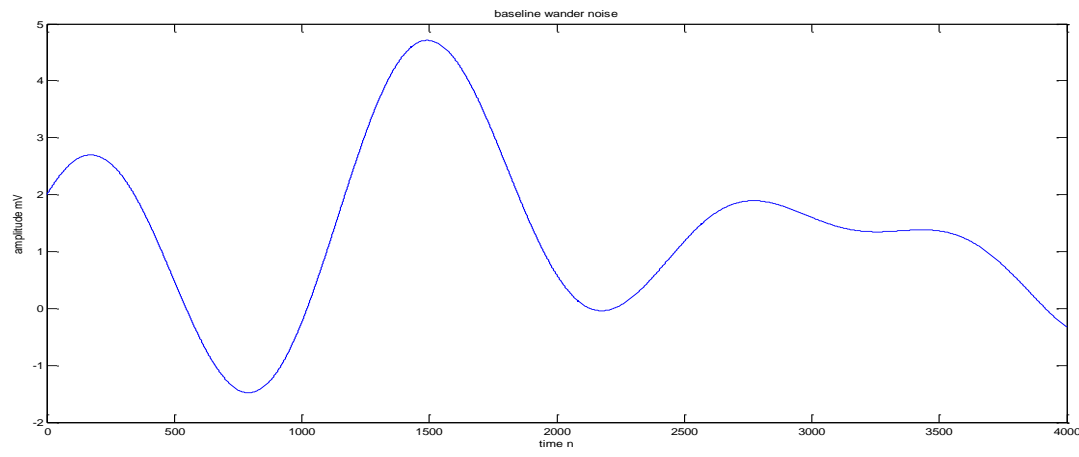

Fig 3 Generation of low frequency noise(baseline wander)

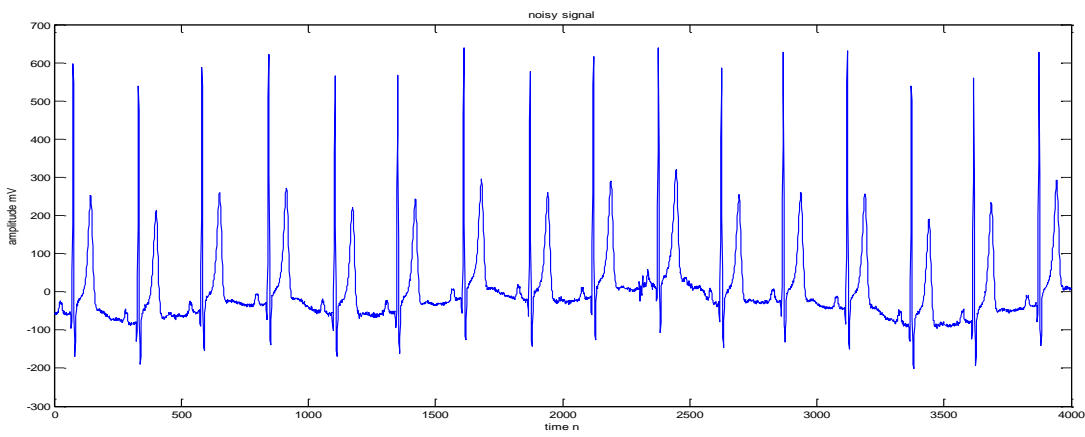

Fig 4. Signal with baseline wander noise added

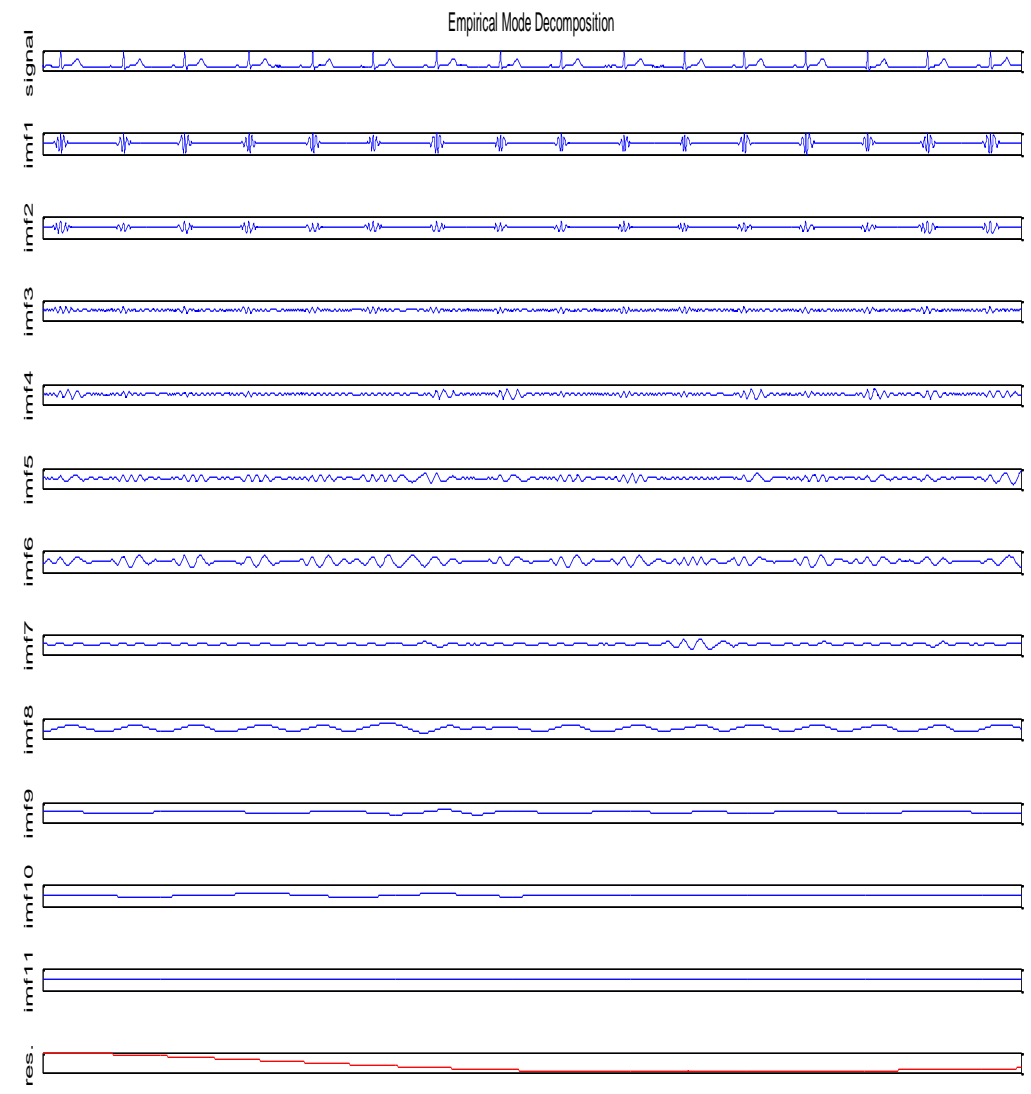

Fig 5. Noisy signal along with its 12 IMFs including residue. 
Baseline wander Removal in ECG using an efficient method of EMD in combination with wavelet.

Table 1 Comparison of EMD-FIR and EMD- Wavelet Method

\begin{tabular}{|c|c|c|}
\hline Record No. & SNR (EMD-FIR) & $\begin{array}{c}\text { SNR (EMD- } \\
\text { WT) }\end{array}$ \\
\hline 100 & 5.03 & 12.05 \\
\hline 103 & 4.71 & 10.07 \\
\hline 105 & 7.88 & 10.13 \\
\hline 119 & 9.33 & 13.02 \\
\hline 120 & 5.59 & 9.76 \\
\hline
\end{tabular}

\section{Conclusion}

This research work throws light on different denoising algorithms used for ECG filtering. The limitations of FIR and IIR filtering methods are mentioned above. The wavelet method or the FIR filtering method can't be used directly because of the limitations mentioned above. However when such methods are combined with EMD, we get improved results. From the final result TABLE 1, we conclude that EMD method used with WT filtering removes the baseline wander noise effectively.

\section{References}

[1]. D.C Reddy. "Biomedical Signal Processing" Pages 18-19

[2]. Emmanuel C. Ifeachor and Barrie W. Jervis, "Digital Signal Processing :A practical approach", 2nd edition, 2009, pearson education Ltd., ISBN: 978-81-317-0824-8.

[3]. N. B. Jones and J. D. McK. Watson, "Digital Signal Processing: Principles, Devices and Applications", 1990 edition, Peter peregrines Ltd., London, UK, ISBN: 0-86341-210-6.

[4]. Mahmoodabadi and S. Ahmadian, "ECG feature extraction based on multiresolution wavelet transform", Proceedings of the IEEE 27th Annual Conference on Engineering in Medicine and Biology, January 2005. pp. 3902-3905, Shanghai, China.

[5]. R.J.E. Merry, "Wavelet Theory and Applications A literature study" Eindhoven University of Technology Department of Mechanical Engineering, June 7, 2005.

[6]. O. Rioul and M. Vetterli, "Wavelets and signal processing”, IEEE Signal Processing Magazine, vol. 8, no. 4, August 1991. pp. 1438 ,

[7]. Stephane G. Mallat, “A theory for multiresolution signal decomposition: The wavelet representation”, IEEE Transactions on Pattern Analysis and Machine Intelligence, Vol. 11 , no. 7, August 1989. pp. 674-693,

[8]. P. Flandrin, P. Goncalves, and G. Rilling, Detrending and denoising with empirical mode decompositions," in EUSIPCO-04, 2004, pp. $1581\{1584$.

[9]. N.E. Huang, Z. Shen, S.R. Long, M.L. Wu, H.H. Shih, Q. Zheng, N.C. Yen, C.C. Tung and H.H. Liu, "The empirical mode decomposition and Hilbert spectrum for nonlinear and nonstationary time series analysis," Proc. Roy. Soc. London A, Vol. 454, 1998. pp. 903-995.

[10]. S. A. Jones, ECG Notes: Interpretation And Management Guide, ser. G - Reference, Information and Interdisciplinary Subjects Series. F.A. Davis, 2005. 